\title{
VARIATION OF CERVICAL CYTOLOGY BY PAP SMEAR AND ITS CORRELATION WITH CERVICAL BIOPSY IN PATIENTS ATTENDING BIRAT MEDICAL COLLEGE TEACHING HOSPITAL, MORANG, NEPAL
}

Amrita Sinha ${ }^{1^{*}}$, Santosh Upadhyaya Kafle ${ }^{2}$, Mrinalini Singh $^{3}$, Neeta Kafle $^{1}$

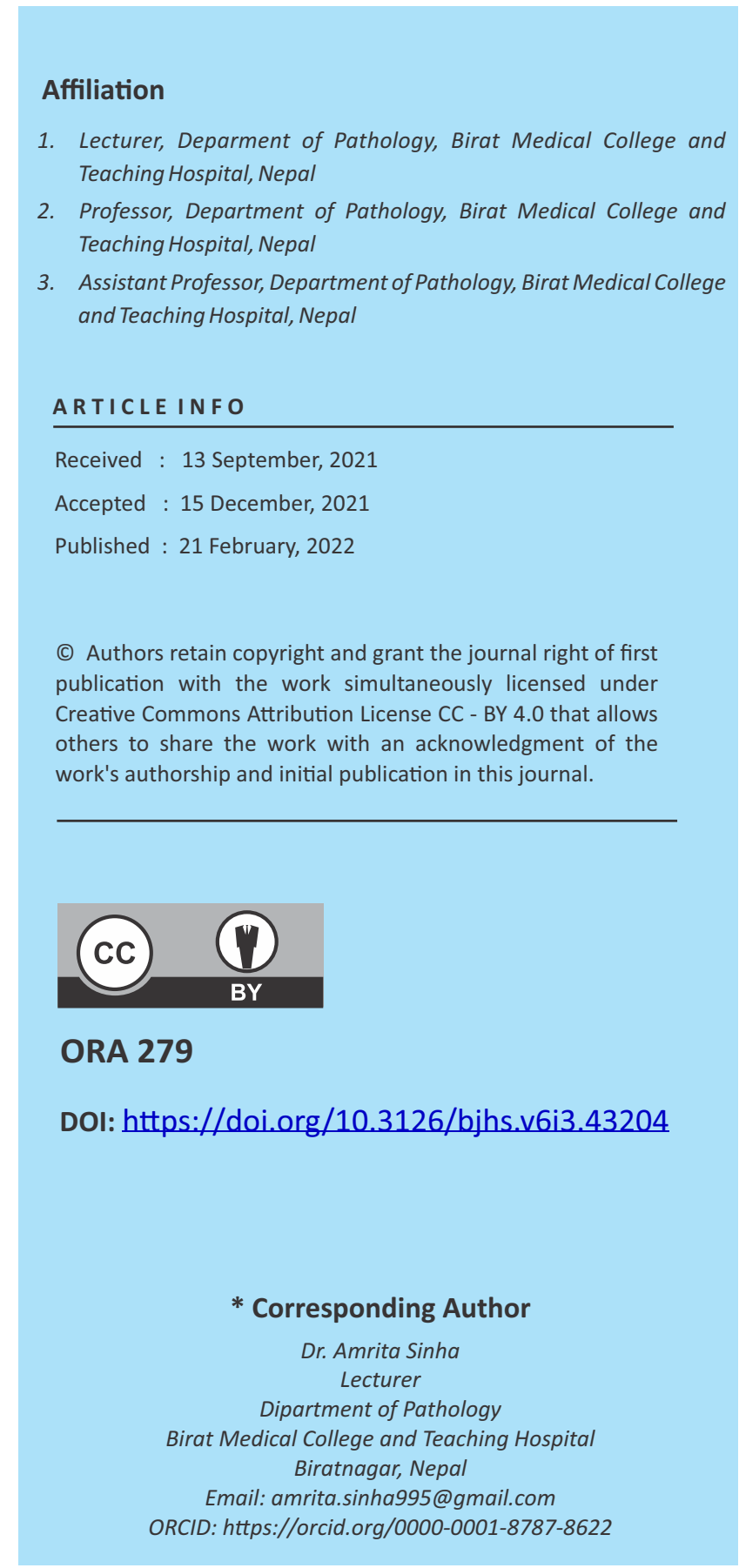

\section{Citation}

Variation of Cervical Cytology by Pap Smear and its Correlation with Cervical Biopsy in Patients Attending Birat Medical College Teaching Hospital, Morang, Nepal. Amrita Sinha, Santosh Upadhyaya Kafle, Mrinalini Singh, Neeta Kafle. BJHS 2021;6(3)16.1615-1620.

\section{ABSTRACT}

\section{Introduction}

Carcinoma cervix is the second most common cause of death in women worldwide and the most common cause in developing countries. Cervical pap smear is a simple, safe, noninvasive, outdoor and low cost effective screening method for detection of cervical lesion but has limitations and needs confirmation by histopathology. Cervical biopsy is a gold standard but invasive technique.

\section{Objectives}

The main objective of this study is to find the changes of cervical cytology by pap smear and to correlate the changes observed in cervical cytology with cervical biopsy.

\section{Methodology}

This is a hospital based prospective cross-sectional study carried out from September 15, 2020 to May 31, 2021 in Birat Medical College where all pap smears received in the Department of Pathology were included in the study. The cases that had undergone both pap smear and cervical biopsy were compared. The correlation was then done regarding cytological and histological diagnosis.

\section{Result}

In this study, total 550 pap smears were performed and out of them 30 patients were advised for cervical biopsy. Majority of patients were in the age group of 31-40 (38.5\%) years. The pap smear findings revealed $93.4 \%$ as negative for intra epithelial lesion or malignancy (NILM) and $6.5 \%$ as epithelial cell abnormality (ECA). ECA comprised atypical squamous cells of undetermined significance (ASCUS) with $3.6 \%$, low-grade squamous intra epithelial lesion with $1.6 \%$, and high-grade squamous intra epithelial lesion with $0.9 \%$. There were two cases $(0.4 \%)$ of malignancy. Sensitivity, specificity and positive predictive value of Pap smear were $90.9 \%, 89.5 \%$, and $83.3 \%$ respectively.

\section{Conclusion}

Pap smear is a cost effective screening method for early detection of premalignant and malignant cervical lesions. However, biopsy is considered to be the gold standard for the confirmation of abnormalities detected in cervical smear provided that it is taken from the representative areas.

\section{KEYWORDS}

Cervix, Female, Pap smear 


\section{INTRODUCTION}

Carcinoma cervix is the second most common cause of death in women worldwide and the most common cause in developing countries. ${ }^{1}$ It has been considered preventable because it has a long pre-invasive state and the availability of screening programs and treatment of pre-invasive lesion is effective. ${ }^{2}$ The pre-invasive stages consists of cellular alterations in the cervix which compromise the epithelium of the region and translate into cervical intraepithelial neoplasia (CIN), divided into grades I, II and III (carcinoma in situ). ${ }^{3}$

The premalignant lesions of cervix commonly involve transformation zone of the uterine cervix. Histopathological terminologies used to describe the grades of the disease are: low grade CIN (cervical intraepithelial neoplasia) comprises CIN I lesions with koilocytic atypia and, high grade CIN consist of CIN II and III. In histopathology, mild dysplasia (CIN I) corresponds to low-grade squamous intraepithelial lesions (LSIL) in cytology whereas moderate and severe dysplasia (CIN II and CIN III) corresponds to high grade intraepithelial lesions (HSIL) in cytology. High grade lesions are true precursors of invasive cancer. ${ }^{4}$

The exfoliative cytology has proven to be an efficacious technique in detection of cancerous and precancerous lesions of the cervix..$^{5}$ The Papanicolaou (Pap) smear is a simple, safe, noninvasive and low cost effective method for detection of precancerous, cancerous and non-cancerous changes in the cervix. ${ }^{6}$ The cytology offers certain advantages over histology in the assessment of cervical dysplasia. It provides exquisite nuclear detail, making early nuclear abnormalities easier to appreciate. Broad area is sampled by a good Pap collection, where only a portion of the squamocolumnar junction may be sampled by biopsy. However, pap smear fails to localize the lesion and even though pap smear has become a standard screening test, cervical biopsy remains the 'gold standard' for the diagnosis of precancerous cervical lesion. ${ }^{8}$

Abnormal cervical pathology are common findings. Sometimes, a diagnostic dilemma occurs which can be minimized after having cervical biopsy. So we need to find correlation between cervical cytology and biopsy in our settings. This type of study has been done by Dhakal et al $^{4}$ in Nepal and Bamanikar SA et al $^{11}$ in India however not done in our setup so this study is done in our settings. The general objective of this study is to find the changes of cervical cytology by pap smear and the specific objective is to correlate the changes observed in cervical cytology with cervical biopsy.

\section{METHODOLOGY}

This is a hospital based prospective cross-sectional study carried out from $15^{\text {th }}$ September 2020 to $31^{\text {st }}$ May 2021 in the department of Pathology, Birat Medical College and Teaching Hospital. Ethical clearance was obtained from the Institutional Review Committee (IRC) of the institute to carry out the study. The informed consent was taken from the patients. All samples requested for pap-smear were studied during this period. In total 550 samples were studied. However, the cases who had undergone both pap-smear and cervical biopsy were compared. Papanicolaou's method was used for staining pap smears. The new 2014 Bethesda system was used for cytological interpretation of the smears. The cervical biopsies were fixed in $10 \%$ formalin, processed and stained with Haematoxylin and Eosin stain for histopathological examination. The data were entered into Microsoft Excel and then transferred to statistical package of social science (SPSS) version 16 for the analysis. Frequencies and percentages were used to present the data. Sensitivity, specificity and positive predictive value were calculated. Women $>65$ years and $<20$ years, those female with frank cancer, pregnant women, post total hysterectomy patient and smears unsatisfactory for evaluation were excluded from the study.

\section{RESULTS}

A total of 550 cervical pap smears were received in the Department of Pathology. Age of the patients ranged from 21 to 78 years with the majority of cases belonging to the age group of 31-40 years.

On cytology, total 514(93.4\%) cases were negative for intra epithelial lesion or malignancy (NILM), 329 (59.8\%) were normal smear and $185(33.6 \%)$ were inflammatory smears. Total $36(6.5 \%)$ cases were squamous epithelial cell abnormalities (ECA), 20(3.6\%) were atypical squamous cells of undetermined significance (ASCUS), 9 (1.6\%) were LSIL, 5 $(0.9 \%)$ were $\mathrm{HSIL}$ and $2(0.4 \%)$ were Squamous cell carcinoma (SCC). (Table 1)

\begin{tabular}{|c|c|c|c|c|c|c|c|}
\hline \multirow[b]{2}{*}{$\begin{array}{c}\text { Age group } \\
\text { (years) }\end{array}$} & \multicolumn{2}{|c|}{ NILM } & \multicolumn{4}{|c|}{ ECA : Squamous } & \multirow[b]{2}{*}{ Total } \\
\hline & $\begin{array}{c}\text { Normal } \\
\text { smear }\end{array}$ & $\begin{array}{c}\text { Inflammatory } \\
\text { smear }\end{array}$ & ASCUS & LSIL & HSIL & SCC & \\
\hline $21-30$ & 97 (29.5\%) & 35 (18.9\%) & $0(0 \%)$ & 0 & 0 & 0 & $132(24.0 \%)$ \\
\hline $31-40$ & $140(42.6 \%)$ & 69 (37.3\%) & $1(5.0 \%)$ & $2(22.2 \%)$ & 0 & 0 & $212(38.5 \%)$ \\
\hline $41-50$ & $73(22.2 \%)$ & $63(34.1 \%)$ & $14(70.0 \%)$ & $1(11.1 \%)$ & 0 & 0 & $151(27.5 \%)$ \\
\hline $51-60$ & $16(4.9 \%)$ & $11(5.9 \%)$ & $4(20.0 \%)$ & $6(66.7 \%)$ & $3(60.0 \%)$ & $1(50.0 \%)$ & $41(7.5 \%)$ \\
\hline $61-70$ & $2(0.6 \%)$ & $3(1.6 \%)$ & $1(5.0 \%)$ & 0 & $1(20.0 \%)$ & $1(50.0 \%)$ & $8(1.5 \%)$ \\
\hline$>70$ & $1(0.3 \%)$ & $4(2.2 \%)$ & 0 & 0 & $1(20.0 \%)$ & 0 & $6(1.1 \%)$ \\
\hline Total & $329(59.8 \%)$ & $185(33.6 \%)$ & $20(3.6 \%)$ & $9(1.6 \%)$ & $5(0.9 \%)$ & $2(0.4 \%)$ & 550 \\
\hline
\end{tabular}


Thirty cases had both pap smear and cervical biopsy. In the biopsy findings, Chronic cervicitis was the most common finding which comprised of $18(60.0 \%)$ cases. Normal cases were seen in 1 (3.3\%) biopsies. Cervical intra-epithelial neoplasia I (CIN I), CIN II and CIN III cases were seen in $5(16.7 \%), 1(3.3 \%)$ and $2(6.7 \%)$ biopsies respectively. Malignancy was seen in $3(10.0 \%)$ cases which comprised of Squamous cell carcinoma. (Table 2)

\begin{tabular}{|c|c|c|}
\hline Cervical biopsy & Frequency & $\begin{array}{c}\text { Percentage } \\
\text { (\%) }\end{array}$ \\
\hline Normal & 1 & 3.3 \\
\hline Chronic cervicitis & 18 & 60.0 \\
\hline CINI(Mild dysplasia) & 5 & 16.7 \\
\hline $\begin{array}{l}\text { CINII } \\
\text { (Moderate dysplasia) }\end{array}$ & 1 & 3.3 \\
\hline CINIII (Severe dysplasia) & 2 & 6.7 \\
\hline Malignancy & 3 & 10.0 \\
\hline Total & 30 & 100 \\
\hline
\end{tabular}

In case of inflammatory smear, 16 cases were processed for histopathological examination, 15(83.3\%) were reported as chronic cervicitis and 1 (20.0\%) was reported as CIN I. Out of 20 cases of ASCUS, 2 cases were processed for histopathological examination, 1 (5.6\%) was reported as chronic cervicitis and 1 (20.0\%) was reported as CIN I. In case of LSIL, 5 cases were processed for histopathological examination, 1 (5.6\%) was reported as chronic cervicitis, $3(60.0 \%)$ were reported as CIN I and 1(50.0\%) was reported as CIN III. Similarly, in case of HSIL, 3 cases were processed for histopathological examination, 1 (100.0\%) was reported as CIN II, 1(50.0\%) was reported as CIN III and 1 ((33.3\%) was reported as SCC. (Table 3)
Table 4: $2 * 2$ table of cytology and histology findings for detecting malignancy

\begin{tabular}{|l|l|l|c|}
\hline \multicolumn{4}{|c|}{ Histological Diagnosis } \\
\hline Cytological Diagnosis & $\begin{array}{l}\text { positive for } \\
\text { malignancy }\end{array}$ & $\begin{array}{l}\text { negative for } \\
\text { malignancy }\end{array}$ & Total \\
\hline positive for malignancy & $10(90.9 \%)$ & $2(10.5 \%)$ & $12(40.0 \%)$ \\
\hline $\begin{array}{l}\text { Negative for } \\
\text { malignancy }\end{array}$ & $1(9.1 \%)$ & $17(89.5 \%)$ & $18(60.0 \%)$ \\
\hline Total & 11 & 19 & 30 \\
\hline
\end{tabular}

Chi square value $18.76 \mathrm{P}$ value $=0.000$

Positive predictive value $83.3 \%$

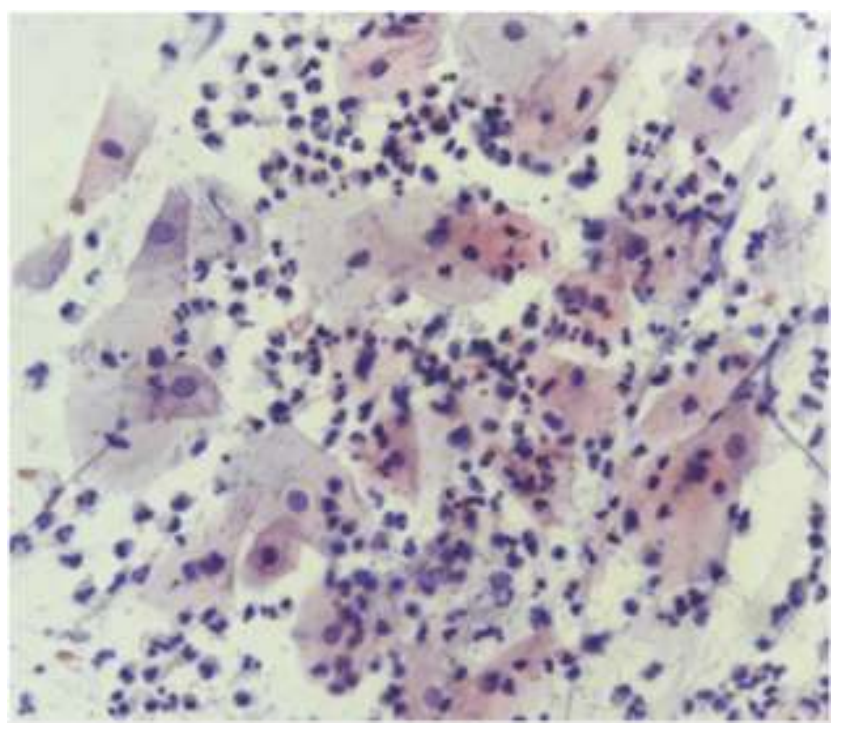

Figure 1: Inflammatory Papanicolaou smear (x400)

Table 3: Correlation between cervical pap smear and cervical biopsies findings
\begin{tabular}{|l|c|c|c|c|c|c|c|}
\hline & & Cervical biopsy findings & & & \\
\hline $\begin{array}{c}\text { Cervical pap } \\
\text { smear findings }\end{array}$ & Normal & $\begin{array}{c}\text { Chronic } \\
\text { cervicitis }\end{array}$ & CINI & CINII & CINIII & Malignancy & Total \\
\hline Normal smear & $1(100.0 \%)$ & $1(5.6 \%)$ & 0 & 0 & 0 & 0 & $2(6.7 \%)$ \\
\hline $\begin{array}{l}\text { Inflammatory } \\
\text { smear }\end{array}$ & 0 & $15(83.3 \%)$ & $1(20.0 \%)$ & 0 & 0 & 0 & $16(53.3 \%)$ \\
\hline ASCUS & 0 & $1(5.6 \%)$ & $1(20.0 \%)$ & 0 & 0 & 0 & $2(6.7 \%)$ \\
\hline LSIL & 0 & $1(5.6 \%)$ & $3(60.0 \%)$ & 0 & $1(50.0 \%)$ & 0 & $5(16.7 \%)$ \\
\hline HSIL & 0 & 0 & 0 & 1 & $1(50.0 \%)$ & $1(33.3 \%)$ & $3(10.0 \%)$ \\
\hline Malignancy & 0 & 0 & 0 & 0 & 0 & $\mathbf{2}(66.7 \%)$ & $2(6.7 \%)$ \\
\hline Total & $\mathbf{1 ( 3 . 3 \% )}$ & $\mathbf{1 8 ( 6 0 . 0 \% )}$ & $\mathbf{5 ( 1 6 . 7 \% )}$ & $\mathbf{1 ( 3 . 3 \% )}$ & $\mathbf{2 ( 6 . 7 \% )}$ & $\mathbf{3 ( 1 0 . 0 \% )}$ & $\mathbf{3 0 ( 1 0 0 \% )}$ \\
\hline
\end{tabular}

The sensitivity, specificity and positive predictive value of pap smear were $90.9 \%, 89.5 \%$ and $83.3 \%$ respectively in our study. (Table 4) 


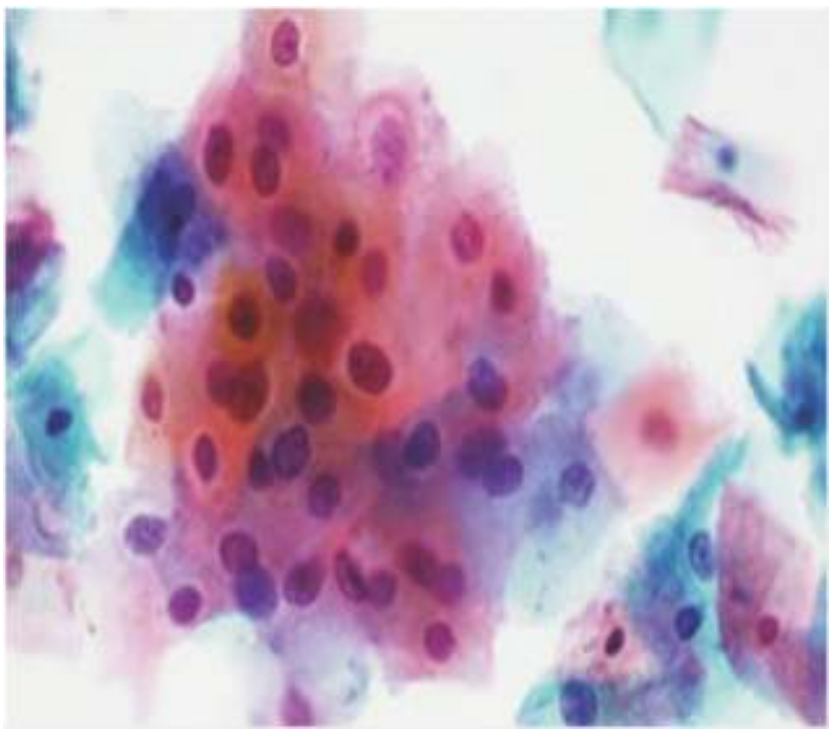

Figure 2: Atypical squamous cells of undetermined significance on Papanicolaou smear (x400)

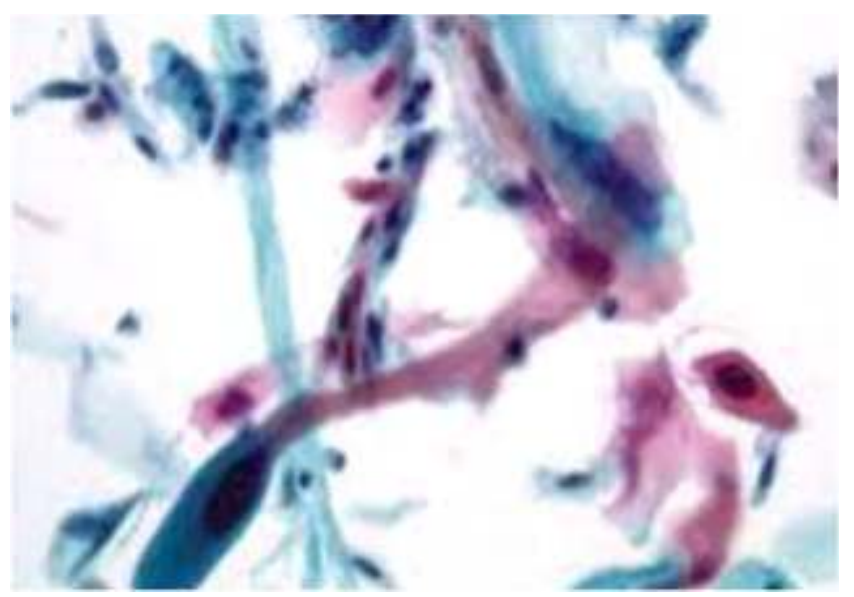

Figure 3: Squamous cell carcinoma on Papanicolaou smear $(x 400)$

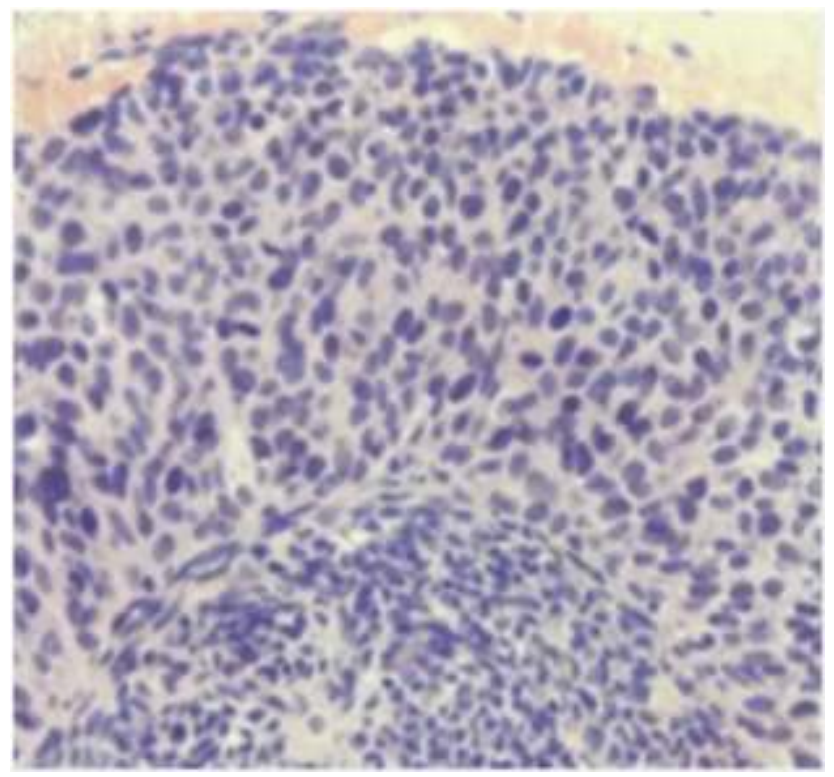

Figure 4: Cervical intraepithelial neoplasia III on $\mathrm{H}$ and $\mathrm{E}$ stain (x400)

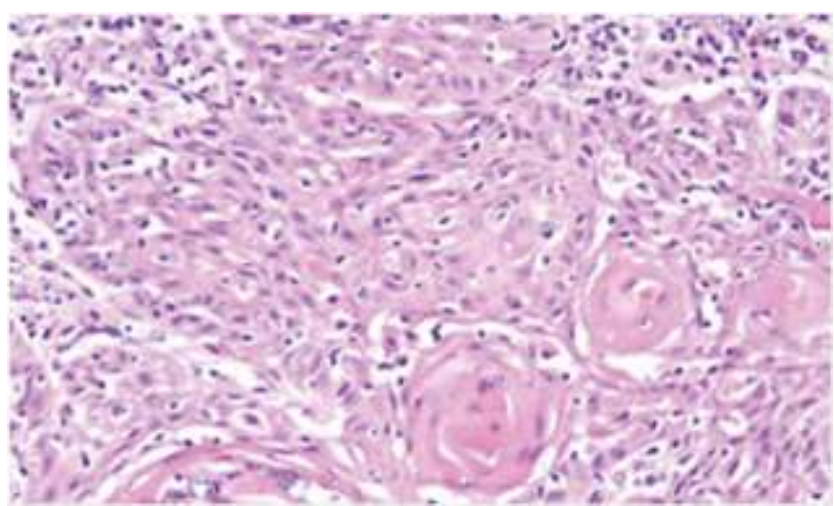

Figure 5: Squamous cell carcinoma on $\mathrm{H}$ and $\mathrm{E}$ stain (x400)

\section{DISCUSSION}

Cervical carcinoma has a long premalignant latent phase, which precedes the invasive disease, can be detected on cytological examination, and are treatable. It is considered to be an ideal gynaecological malignancy for screening as it meets both test and disease criteria for screening. ${ }^{6}$ George Papanicolaou introduced pap smear in 1947, after which it has become the main screening tool for the detection of cervical pathology. ${ }^{8}$ All sexually active women above the age of 18 years should have an yearly pap smear for three consecutive years according to the American Cancer Society, National Cancer Institute American College of Obstetrics and Gynecologists guideline. And if there is three consecutive negative pap smears, the test can be extended for 3-5 years. ${ }^{9,10}$

In this present study, maximum number of patients were in the age group of 31-40 years, which comprised of 212 (38.5\%), followed by 151 (27.5\%) in the age group of $41-50$ years. This was similar to the study done by Dhakal et al (35.4\%), Mainali et al (38.14\%) and Bamanikar et al (35.6\%) where majority of cases were seen in the age group of 31-40 years. ${ }^{4,8,11}$ This indicates that premalignant lesions precedes the development of malignancy by a decade and prompt identification by appropriate screening reduces the mortality and morbidity associated with cervical cancer.

Pap smear cytology findings were categorized into NILM and ECA. In this study, Pap smear reported as NILM was the most common findings with $93.4 \%$ of all smears examined which was in accordance with the study conducted by Bamanikar et al. where $88.02 \%$ cases were reported as NILM. ${ }^{11}$

In our study, among 514 cases reported as NILM, normal smear $(n=329 ; 59.8 \%)$ was the most common observation followed by inflammatory smear $(n=185 ; 33.6 \%)$. The result of our finding was very similar to the finding in a study done by Dhakal et al where $67.9 \%$ were normal smear and $27.9 \%$ were inflammatory smear and Mainali et al where $59.51 \%$ were normal smear and $39.12 \%$ were inflammatory smear. ${ }^{4,8}$ In our study, ECA was found in $6.5 \%$ cases which was in accordance with the study conducted by Bamanikar et alwhere $5.99 \%$ cases were reported as ECA. ${ }^{11}$ ECA group were categorized as ASCUS, LSIL, HSIL and SCC.

In this study, ASCUS, LSIL, HSIL and frank malignancy cases were 20(3.6\%), 9 (1.6\%), $5(0.9 \%)$ and $2(0.4 \%)$ respectively 
which was similar to the study done by Bamanikar et al who reported ASCUS, LSIL, HSIL and frank malignancy in 2.98\%, $1.19 \%, 0.66 \%$ and $0.95 \%$ respectively. ${ }^{11}$ However, findings in the study done by Gandavaram et al revealed ASCUS, LSIL, HSIL and frank malignancy in $13.6 \%, 9.6 \%, 6.4 \%$ and $3.2 \%$ respectively. ${ }^{12}$ These differences in pap smear reporting can be due to the differences in sampling, staining, fixation technique and reporting errors.

On histopathological examination, out of 30 patients, on whom pap smear and biopsy were taken, majority of the cases $(n=18 ; 60.0 \%)$ had chronic cervicitis followed by mild dysplasia $(n=5 ; 16.7 \%)$, moderate to severe dysplasia $(n=3 ; 10 \%)$, malignant lesion $(n=3 ; 10 \%)$ and normal case $(n=1 ; 3.3 \%)$. Findings of our study coincide with the reports of Bamanikar et al who reported chronic cervicitis in 59\% of cases, mild dysplasia in $16.5 \%$ cases, moderate to severe dysplasia in $11.5 \%$ cases, malignant lesion in $10 \%$ cases and normal findings in $3 \%$ cases in his study. ${ }^{11}$

Similar finding was also seen in a study conducted by Gandavaram et al, where chronic cervicitis, mild dysplasia, moderate to severe dysplasia and malignant lesion were reported in $56.8 \%, 19.2 \%, 14.4 \%$ and $9.6 \%$ of cases in his study. $^{12}$

In this present study, out of 2 cases diagnosed as ASCUS on cytology, one was non neoplastic and one was CIN I in histopathology. Fifty percent of ASCUS on cytology revealed the diagnosis of CIN on histopathology stating that all cases of ASCUS should be followed up periodically and biopsy is must in this condition. Out of 5 cases diagnosed as LSIL on cytology, one was non-neoplastic, three were CIN I and one was CIN III on histopathology. The false positive cases of ASCUS and LSIL on pap smear were due to regenerative changes whose evaluation therefore becomes more important. The regenerative changes can be divided into typical and atypical and the atypical regenerative changes should be added to the ASCUS group. Also to decrease false positive rate, adequate sampling and avoiding technical errors like air drying, fixation artefacts is necessary. In this study, out of 3 cases diagnosed as HSIL on cytology, one was CIN II, one was CIN III and 1 case was diagnosed as SCC on biopsy, hence biopsy from representative area would minimize the errors. The chances of HSIL progressing to invasive cancer is only $2 \%$ well documented in literature, however, up to $20 \%$ with HSIL will develop cancer if it is left untreated. Therefore to prevent HSIL from developing into cancer, the precancerous cells are removed or destroyed.

In our study, sensitivity, specificity and positive predictive value of pap smear were $90.9 \%, 89.5 \%$ and $83.3 \%$

\section{REFERENCES}

1. Siddegowda RB, DivyaRaniMN ,Natarajan M, Biligi DS. Inter-observer variation in reporting of pap smears. National J Laboratory Med. 2016;5(3):22-5. DOI: 10.7860/NJLM/2016/20002:2135

2. Joshi C, Kujur P, Thakur N. Correlation of papsmear and colposcopy in relation to histopathologicalfindings in detection of premalignant lesions of cervix in a Tertiary Care Centre.Int J Sci Stud. 2015; 3(8):5560. DOI: $10.17354 / \mathrm{ijss} / 2015 / 508$ respectively which was comparable with Bamanikar et al where sensitivity, specificity and positive predictive value of pap smear were $89.47 \%, 88.70 \%$ and $82.92 \%$ respectively. ${ }^{11}$ Similar findings were reported by Tamboli et al where sensitivity, specificity and positive predictive value of pap smear were $90.65 \%, 90.27 \%$ and $89.81 \%$ respectively and the values reported by Jones et al were $89.4 \%, 64.8 \%$ and $88.9 \%$ respectively. ${ }^{13,14}$

The sensitivity and specificity of pap smear can be increased by adopting proper technique and adequate sampling from the transformation zone. In this study, pap smears were sampled by conventional method and therefore use of liquid based cytology is advised to improve the sensitivity and specificity of pap smear.

\section{CONCLUSION}

Cervical pap smear is a cost-effective and reliable screening test for early detection of premalignant and malignant cervical lesions. Therefore, it should always be done as a routine outpatient screening test as per guidelines. However, cervical biopsy is the gold standard for its confirmation, which should be carried out to confirm the findings of pap smear and in case of strong clinical suspicion.

\section{RECOMMENDATIONS}

In the view of high probability of carcinoma cervix, screening for carcinoma cervix on a regular basis needs to be considered.

\section{LIMITATION OF THE STUDY}

An important limitation to our study is the small number of patients. Definite categorization of inflammatory smear is not done in our study. Special stains and Immuno cytochemistry is not done.

\section{ACKNOWLEDGEMENT}

We highly acknowledge the Department of Community Medicine for helping us in the statistical analysis of data and the technical staff from the department of pathology for their support in processing the samples and restoring patient records during the study period.

\section{CONFLICT OF INTEREST}

None

\section{FINANCIAL DISCLOSURE}

None

3. Anschau F, Goncalves MAG. Discordance between cytology and biopsy. Histology of cervix: What to consider and what to do. ActaCytologica. 2011;55:158-62. DOI: 10.1159/00032091

4. Dhakal R, Makaju R, Sharma S, Bhandari S, Shrestha S, Bastakoti R. Correlation of cervical pap smear with biopsy in the lesion of cervix. Kathmandu Univ Med J. 2016;14:254-7. PMID: 28814689

5. Tritz DM, Weeks JA, Spires SE, et al: Etiologies for non-correlating cervical cytologies and biopsies. Am J ClinPathol 1995; 103: 594-7. DOI: $10.1093 /$ ajcp/103.5.594. 
6. Bodal VK, Bal MS, kaur B, Kaur S, Suri AK, Bhagat R, Geetanjali. Correlation of papsmear with histopathologicalfindings in malignant and non malignantlesions of cervix. Global Journal of Medical Research 2014;14(4):19-24.

7. Al-Mosawi FH. Comparativestudyofpapsmear and cervical biopsy findings. Kerbala Journal of Medicine.2015;8 (2):2272-81.

8. Mainali, N., Homagai, N., Nepal, N., \&Choudhary, P. A correlation study of cervical cytology on Pap smear with cervical biopsy in a tertiary hospital of Eastern Nepal. J PathologyofNepal.2018: 8(2);1389-92. https://doi.org/10.3126/jpn.v8i2.20993

9. Mettlin C, Dodd GD. The American Cancer Society Guidelines for the cancer-related checkup: an update. CA Cancer J Clin. 1991;41:279-82. DOI: 10.3322/canjclin.41.5.279

10. ACOG committee opinion. Routine cancer screening. Int Jgynaecoo bstet:. 1997;59(2):157-61. PMID: 9431884.
11. Bamanikar SA, Baravkar D, Chandanwale S, Dharwadkar A, Paranjape S. Study of cervical cytology and its correlation with clinical and histopathological findings. Clin Cancer Investig J 2016;5:403-8. DOI: 10.4103/2278-0513.197869.

12. Gandavaram J, PamulapatiBR.Correlation of pap smear and colposcopic findings in relation to histopathological findings among women attending a tertiary care hospital: a two year study. Int J ReprodContraceptObstet Gynecol.2019;8(6):2163-8. DOI: http:// dx.doi.org/10.18203/2320-1770.ijrcog20192132.

13. Tamboli GD, Khatod LV. Accuracy of cytological findings in abnormal cervical smear by cyto-histological comparison. J Med Educ Res 2013;3:19-24.

14. Jones BA, Novis DA. Cervical biopsy-cytology correlation.A College of American Pathologists Q-probes study of 22439 correlations in 348 laboratories. Arch Pathol Lab Med 1996;120(6):523-31. PMID: 8651852. 